\title{
Erratum to: Bioresorbable distraction device for the treatment of airway problems for infants with Robin sequence
}

\author{
Corstiaan C. Breugem ${ }^{1,2} \cdot$ Emma C. Paes $^{1} \cdot$ Moshe Kon $^{1} \cdot$ Aebele B. Mink van der \\ Molen ${ }^{1}$
}

Published online: 10 July 2015

(C) Springer-Verlag Berlin Heidelberg 2015

Erratum to: Clinical Oral Investigations 16 (4) : 1325-1331

\section{DOI 10.1007/s00784-011-0624-x}

The original version of the above article contained a mistake in the presentation of the name Aebele B. Mink van der Molen. The family name should be Mink van der Molen and not van der Molen alone. Also, Corstiaan Breugem should be Corstiaan C. Breugem.

1 Cleft Palate Team, Department of Pediatric Plastic Surgery, Wilhelmina Children's Hospital, P.O. Box 85090, 3508

AB Utrecht, The Netherlands

2 Meander Medical Centre, P.O. Box 1502, 3800

BM Amersfoort, The Netherlands 